\title{
Pulsed and narrowband mixed interference mitigation technique for single antenna GNSS receivers
}

\author{
Shumin Huo ${ }^{\text {a)}}$, Junwei Nie, Xiaomei Tang, \\ Zhe Liu, and Feixue Wang \\ The School of Electronic Science and Engineering, National University of Defense \\ Technology, Changsha 410073, China
}

a)huoshumin123@126.com

\begin{abstract}
Most previous works focus on single type of interference mitigation and simplified combination of these existed methods leads to high disabled probabilities of single antenna Global Navigation Satellite System (GNSS) receivers under mixed interference scenarios due to the interaction of different types of interferers. In this paper, depending on the assumption that pulsed interference repetition period is known, we introduce a segementation method which will guarantee the success of narrowband interference (NBI) power spectrum density (PSD) estimation by minimum operation in mixed interference. Then NBI and pulsed interference can be mitigated, subsequently. The effectiveness is validated by numerical simulation results. Keywords: anti-jamming receiver, Global Navigation Satellite System (GNSS), interference mitigation, pulse blanking (PB)
\end{abstract}

Classification: Navigation, Guidance and Control Systems

\section{References}

[1] P. W. Ward, J. W. Betz, and C. J. Hegarty, "Interference, multipath, and scintillation," in Understanding GPS Principles and Applications, Chaper 6, pp. 243-247, Artech House, Norwood, MA, USA, 2006.

[2] J. Grabowski and C. Hegarty, "Characterization of L5 receiver performance using digital pulse blanking," Proc. of The Institute of Navigation GPS Meeting, Portland, OR, September 2002.

[3] T. Capozza, B. J. Holland, T. M. Hopkinson, and R. L. Landrau, "Single-chip narrow-band frequency-domain excisor for a Global Positioning System (GPS) receiver," IEEE J. Solid-State Circuits, vol. 35, pp. 401-411, Mar. 2000. DOI: $10.1109 / 4.826823$

[4] S. Aromaa, P. Henttu, and M. Juntti, "Transform-selective interference suppression algorithm for spread-spectrum communications," IEEE Signal Process. Lett., vol. 12, no. 1, pp. 49-51, Jan. 2005. DOI:10.1109/LSP.2004.839703

[5] O. A. Yeste Ojeda, J. Grajal, and G. López-Risueno, "Analytical performance of GNSS receivers using interference mitigation techniques," IEEE Trans. Aerosp. Electron. Syst., vol. 49, no. 2, pp. 885-906, April 2013. DOI:10.1109/TAES. 2013.6494387 
continuous and pulsed radio interference with GNSS antenna arrays," International Technical Meeting of the Satellite Division, Savannah, GA, September 2008.

[7] H. Saarnisaari, "Consecutive mean excision algorithms in narrowband or short time interference mitigation," Proc. Position Location and Navigation Symp., Monterey, CA, vol. 1, pp. 447-454, Apr. 2004. DOI:10.1109/PLANS.2004. 1309028

[8] G. X. Gao, "DME/TACAN interference and its mitigation in L5/E5 bands," ION GNSS Conference, Fort Worth, TX, 2007.

[9] E. Anyaegbu, G. Brodin, J. Cooper, E. Aguado, and S. Boussakta, "An Integrated Pulsed Interference Mitigation for GNSS Receivers," J. Navig., vol. 61, pp. 239-255, 2008. DOI:10.1017/S0373463307004572

\section{Introduction}

Periodic-pulsed interference (PPI) and narrowband interference (NBI) are two types of detrimental interference for Global Navigation Satellite System (GNSS) receivers [1] and the immunity of single antenna GNSS receivers against PPI and NBI can be greatly enhanced by the corresponding mitigation techniques $[2,3,4]$. The typically PPI sources for GNSS receivers are transmitted from DME/TACAN, primary L-band radars, AEW\&C L-band radars and TADIL-J/Link-16 [5]. NBIs could originate from amplitude modulation, frequency modulation stations transmitters harmonics or intentional continuous wave (CW) jammers, etc. [1]. Antenna arrays provide an effective way to mitigate mixed interference [6] but are often quite expensive, computationally very demanding and have large size which make the arrays can not be used in every receiver [7]. Besides, it is difficult to combat the mixed interference effectively by simple combination with these conventional mitigation techniques for single antenna GNSS receivers as shown in Section 4 and surprisingly to the best of our knowledge, the mitigation for mixture of PPI and NBI has not received much attention.

For simplicity, only PPIs originating from the primary L-band radars and NBIs whose bandwidth are lower than $10 \%$ of the bandwidth of the desired signal [7] are considered in this paper. The results discussed in the following section for primary L-band radars is easily extended to other cases which is out of the scope of this paper. For primary L-band radars which can be deemed as low duty cycle (DC) PPIs, pulse blanking (PB) [2] is an effective mitigation technique due to its simplicity and effectiveness. Noting that transformed-domain techniques [8,9] can be used to suppress high DC PPIs instead of PB.

\section{Signal model}

The input samples denoted as $x(n)$ with sampling frequency $f_{s}=1 / T_{s}$, can be expressed with

$$
x(n)=s(n)+q(n)+p(n)+\eta(n)
$$

where $s(n)$ denotes the desired GNSS signal, $q(n)$ is the stationary NBI, $p(n)$ is the PPI, and $\eta(n)$ denotes the complex Gaussian white noise with zero mean and 
variance $\sigma_{\eta}^{2}$, i.e., $\eta(n) \sim \mathcal{C N}\left(0, \sigma_{\eta}^{2}\right)$ with $\operatorname{PSD} \mathcal{S}^{\eta}(f)=\sigma_{\eta}^{2} / f_{s}, f \in[-1 / 2,1 / 2)$. In this paper, we assume that all the components in (1) are statistically uncorrelated with each other. Generally, $s(n)$ with PSD $\mathcal{S}^{s}(f)$ and Binary phase shift keying (BPSK) modulation in (1) can be defined as $s(n)=\sqrt{C} d(n) c(n) \exp \left(j 2 \pi f_{d} n+\phi_{0}\right)$, where $C$ is the power, $d(n) \in\{ \pm 1\}$ represents the data modulation, $c(n) \in\{ \pm 1\}$ is the spreading code with chipping rate $R_{c}, j=\sqrt{-1}$ is the imaginary unit, $f_{d} \in[-1 / 2,1 / 2)$ is the Doppler frequency and $\phi_{0}$ is the initial carrier phase. For the sake of clarity, the data modulation term is omitted in the following analyses. This result discussed in the following section based on BPSK signal is easily extended to Binary Offset Carrier (BOC) cases.

The NBI signal $q(n)$ is modeled as a block spectrum $\mathcal{S}^{q}(f)$ with bandwidth $\Delta f \in(0,1)$. It is written as

$$
\mathcal{S}^{q}(f)= \begin{cases}\frac{P_{1}}{\Delta f}, & \left|f-f_{q}\right| \leq \frac{\Delta f}{2} \\ 0, & \text { otherwise }\end{cases}
$$

where $P_{1}, f_{q}$ denote the NBI power and center frequency, respectively. This means that $q(n)$ can be realized by passing a complex white Gaussian process through a band-limited filter and we will generate $q(n)$ by this mean in Section IV. When the narrowband interference is centered on a local maximum in the desired signal spectrum, it causes a local minimum in the effective carrier-to-noise ratio $\left(C / N_{0}\right)$ [1], thus we assume that $f_{q}=f_{d}$ hereinafter. The interference-to-noise (INR) of $q(n)$, denoted as $\mathrm{INR}_{1}$, can be defined as $\mathrm{INR}_{1}=P_{1} / \sigma_{\eta}^{2}$, and we define

$$
\mathcal{S}^{\mathrm{NBI}}(f)=\mathcal{S}^{s}(f)+\mathcal{S}^{q}(f)+\mathcal{S}^{\eta}(f)
$$

as the NBI PSD without PPI disturbance. The $p(n)$ in (1), can assume different forms depending on the type of interference source and can, in general, be represented as

$$
p(n)=i_{c}\left(n T_{s}\right) \sum_{l_{1}=0}^{\infty} p_{0}\left(n T_{s}-l_{1} T_{p}-T_{0}\right)
$$

where $T_{p}$ is the pulse period, $T_{0}$ accounts for an arbitrary time origin, $i_{c}(n)$ is a continuous component and $p_{0}$ is a function defined as

$$
p_{0}(t)= \begin{cases}1, & 0 \leq t \leq \rho T_{p} \\ 0, & \text { otherwise }\end{cases}
$$

where $T_{p}$ is the repetition period, $\rho$ denotes DC, and $T_{0}$ accounts for an arbitrary time origin. In this paper, $i_{c}\left(n T_{s}\right)$ is assumed to be a zero mean complex Gaussian noise interference with power $P_{2}$, which has a bandwidth equal to useful GNSS signals. Thus the INR of $p(n)$, denoted as $\mathrm{INR}_{2}$, can be defined as $\mathrm{INR}_{2}=\rho P_{2} / \sigma_{\eta}^{2}$.

If $\mathcal{S}^{\mathrm{NBI}}$ can be estimated accurately, the pulsed and narrowband mixed interference can be suppressed easily. However, it is difficult to estimate the NBI PSD with traditional consecutive blocks method due to the following reasons. The presence of PPI will make the NBI and pulsed interference blanking weights biased. Even if we know the pulse period and duty cycle, it is still difficult to estimate $\mathcal{S}_{x}^{\mathrm{NBI}}$ due to the uncertainty of $T_{0}$ in (4). In addition, the relation between $P_{1}$ and $P_{2}$ is not assumed. All mentioned above reasons will make the simplified 
combinations of convention interference mitigation techniques failed under the mixed interference scenarios. The proposed technique will be illustrated in Section 3, which can estimate accurately the NBI-only PSD from the mixed interference.

As defined in (2), the $q(n)$ is assumed to be stationary and has a box spectrum of bandwidth $\Delta f$. Thus, the ideal frequency- and time-domain blanking functions, corresponding $q(n)$ and $p(n)$, can be written as

$$
\begin{gathered}
F_{b}(f)= \begin{cases}0, & \left|f-f_{q}\right| \leq \frac{\Delta f}{2} \\
1, & \text { otherwise }\end{cases} \\
T_{b}(n)=1-\sum_{l_{1}=0}^{\infty} p_{0}\left(n-l_{1} T_{p}-T_{0}\right)
\end{gathered}
$$

Based on interference-free signal $x_{0}(n)(=s(n)+\eta(n))$, because it is needless to show any consideration for the effect of interference leakage after mitigation in reality. Based on (6) and (7), the ideal mitigation outputs which are used to evaluate interference mitigation technique performance, can be written as

$$
y(n)=\operatorname{IDFT}\left\{\operatorname{DFT}\left\{x_{0}\right\} \times F_{b}\right\}(n) \times T_{b}(n)
$$

where $y(n)$ is corresponding to the presence of $q(n)+p(n)$ scenarios as shown in Section 4.

\section{Proposed technique}

When the pulsed and narrowband mixed interference is present, all the samples are nearly contaminated by NBI but partial by PPI per pulse period. The key idea of the proposed technique is to estimate the NBI PSD using different blocks per pulse period in which at least one block is not corrupted by the pulsed interference. We assume that the samples of a pulse period are grouped into $L$ blocks with equal block size and the $l$ th block vector of $m$ th pulse period is give by

$$
\mathbf{x}_{m, l}=\left[x\left(0+A_{l}+B_{m}\right), \cdots, x\left(N-1+A_{l}+B_{m}\right)\right]^{T}
$$

where $m=0,1, \cdots ; l=0,1, \cdots, L-1,\lfloor\cdot\rfloor$ rounds the element to the nearest integers toward minus infinity and $A_{l}=\left\lfloor l f_{s} T_{p} / L\right\rfloor, B_{m}=\left\lfloor m f_{s} T_{p}\right\rfloor$.

Based on the idea of the proposed technique, it is significantly to decide how many blocks being segmented per pulse period. Obviously, at least a whole block should not be contaminated by the PPI for $m$ th pulse period. The $m$ th pulse period samples is segmented into $L$ blocks with block size $\left\lfloor f_{s} T_{p} / L\right\rfloor$. Based on the Pigeon Hole principle, if the following expression holds:

$$
f_{s}(1-\rho) T_{p} \geq 2 \times f_{s} T_{p} / L
$$

at least one noncorrupted block by the pulsed interference can be guaranteed. Considering $L$ as a positive integer, (10) can be rewritten as $L \geq\lceil 2 /(1-\rho)\rceil$, where $\lceil\cdot\rceil$ rounds the element to the nearest integers toward infinity. We can choose a data frame with $N$ continuous samples for every block for PSD estimation and $L$ averaged PSDs can be computed over $M$ pulse periods, separately. For the case of $f_{s}=4.092 \mathrm{MHz}, T_{p}^{\min }=1 \mathrm{~ms}, T_{p}^{\max }=2.5 \mathrm{~ms}$ and $\rho^{\max }=10 \%$ for primary Lband radars [5], we can set $N=1024$ and $L=3$, for example. 
Passing through windowing function $w$ of length $N$ and an $N$-point FFT transform ( $N$ is assumed an even number in the letter), the $\mathbf{x}_{m, l}$ yields the Fourier coefficients records $X_{m, l}^{w}(k)=\frac{1}{\sqrt{N}} \sum_{i=0}^{N-1} w(i) \mathbf{x}_{m, l}(i) \exp (-j 2 \pi i k / N), k=-N / 2, \ldots$, $N / 2-1$. We denote the squared magnitude of $X_{m, l}^{w}(k)$ by $\mathcal{S}_{m, l}^{w, x}(k)$, i.e., $\mathcal{S}_{m, l}^{w, x}(k)=\left|X_{m, l}^{w}(k)\right|^{2}$. The $k$ th averaged squared magnitude of Fourier coefficient record for all lth blocks, denoted as $\hat{\mathcal{S}}_{m, l, M}^{w, x}(k)$, can written as $\hat{\mathcal{S}}_{m, l, M}^{w, x}(k)=1 / M \times$ $\sum_{m_{0}=m-M+1}^{m} \mathcal{S}_{m_{0}, l}^{w, x}(k)$. We can choose the minimum value under proper value of $M$ among $L$ averaged PSD blocks at $k$ th frequency bin, i.e.

$$
\min _{l}\left\{\hat{\mathcal{S}}_{m, l, M}^{w, x}(k)\right\}
$$

for windowed NBI PSD estimation due to

$$
\min _{l}\left\{\lim _{M \rightarrow \infty} \mathrm{E}\left\{\hat{\mathcal{S}}_{m, l, M}^{w, x}(k)\right\}\right\}=\sigma_{w}^{2} \mathcal{S}_{x}^{\mathrm{NBI}}(k / N)
$$

where $\sigma_{w}^{2}=\sum_{i=0}^{N-1} w^{2}(i)$ is the energy of the window function $w$.

\section{Numerical results}

The effectiveness of proposed technique are evaluated by means of Monte Carlo simulations. The sampling frequency is fixed at $4.092 \mathrm{MHz}$. A GNSS signal $s(n)$, with signal level of $C / N_{0}=40 \mathrm{~dB}-\mathrm{Hz}, R_{c}=1.023 \mathrm{MHz}$, code length 1023 and $f_{d}=0 \mathrm{rad}$, is selected. The $q(n)$, with bandwidth of $\Delta f=0.05$, and $p(n)$ with maximum fixed DC $\rho=10 \%$ and $T_{p}=1.7 \mathrm{~ms}^{1}$ which is selected between $1 \mathrm{~ms}$ and $2.5 \mathrm{~ms}$ for L-band radars, are considered. The arbitrary time origin $T_{0}$ is drawn uniformly taken from the interval $\left[0, T_{p}\right]$ in each simulation run. The sum of $\mathrm{INR}_{1}$ and $\mathrm{INR}_{2}$ is fixed approximatively at $23 \mathrm{~dB}$ with different $\mathrm{INR}_{1} / \mathrm{INR}_{2}=$ $\{-10,0,10\} \mathrm{dB}$ in order to maintain the NBI and PPI strong enough. In all simulation scenarios, $N=1024, L=4$ and $M=50$ are chosen, respectively. A Hamming window function for the $50 \%$ overlap-and-add approach is used. The scaling factors for NBI and PPI mitigation are set to 4.6 and 5, respectively, which need further optimal investigation. We employ $10^{5}$ correlation values to obtain each point of the ROC curves with a correlation value consisting of coherent integration over $1 \mathrm{~ms}$ duration and a noncoherent accumulation. The synchronization parameters estimations are set as the true values to show the performance of the proposed technique without signal synchronization errors. 'Ideal mitigation' curve is obtained based on (8) and 'Conventional' cases have been obtained by cascading NBI and PPI mitigation techniques.

As depicted in Fig. 1, the proposed technique have achieved better ROC performance compared with conventional method against strong mixed interference. When the ratio changes between $\mathrm{INR}_{1}$ and $\mathrm{INR}_{2}$, the ROC performance of the conventional one fluctuates greatly due to time-varying nature of pulsed interference while the proposed technique presents a robustness to it. Specially, when the ratio equal to $-10 \mathrm{~dB}$ which means that the pulse interference power is ten times of the NBI, the conventional method is failed to suppress the mixed interferences while the proposed technique gives remarkably performance improvements. The

${ }^{1}$ The ROC comparison results of other NBI bandwidths and PPI periods are omitted due to having similar results. 


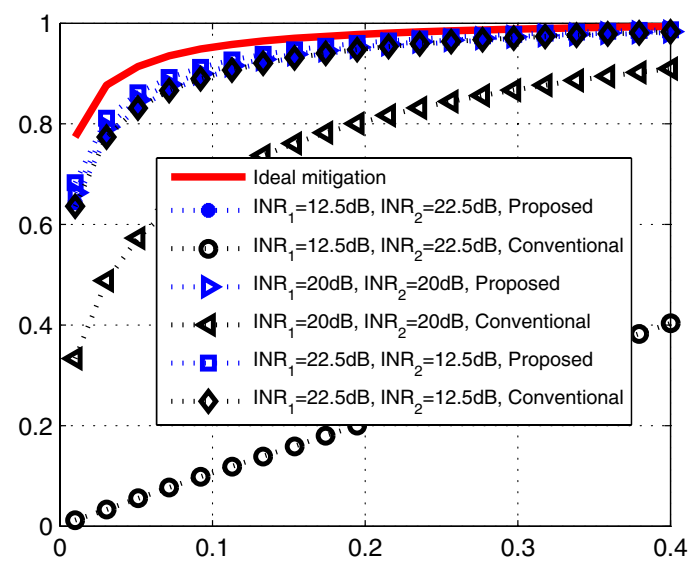

Fig. 1. ROC curve comparisons for mixed interference scenarios with different INR combinations

slight degression for proposed technique compared with the ideal mitigation ROC curves for different INR combinations are due to NBI and PPI blanking ratios increasing and interference leakages under strong interference scenarios.

\section{Conclusion}

In this paper, we proposed a pulsed and narrowband mixed interference mitigation technique for single antenna GNSS receivers. We utilized the segmenting and minimizing operations to estimate the NBI PSD and NBI mitigation follows. Then the residual strong PPI can be suppressed easily. Simulation results show that the proposed technique can suppress the mixed interference effectively while withholding a performance very close to ideal mitigation for strong mixed interference. The reason for achieved performance comes at the cost of a prior knowledge of pulse repetition period and the stationary assumption of NBI. Future investigations will be focused on the no priori information about the pulsed interference period scenarios. 